\title{
Prognostic and therapeutic relevance of FLIP and procaspase-8 overexpression in non-small cell lung cancer
}

\author{
JS Riley ${ }^{1}$, R Hutchinson ${ }^{1}$, DG McArt ${ }^{1}, \mathrm{~N}$ Crawford $^{1}$, C Holohan ${ }^{1}$, I Paul ${ }^{1}$, S Van Schaeybroeck ${ }^{1}$, M Salto-Tellez ${ }^{1}$, PG Johnston ${ }^{1}$, \\ DA Fennell ${ }^{2}, K_{\text {Gately }}{ }^{3}, \mathrm{~K}^{1}$ 'Byrne $^{3}$, R Cummins ${ }^{4}$, E Kay ${ }^{4}$, P Hamilton ${ }^{1}$, I Stasik ${ }^{1}$ and DB Longley ${ }^{\star, 1}$
}

Non-small cell lung carcinoma remains by far the leading cause of cancer-related deaths worldwide. Overexpression of FLIP, which blocks the extrinsic apoptotic pathway by inhibiting caspase-8 activation, has been identified in various cancers. We investigated FLIP and procaspase-8 expression in NSCLC and the effect of HDAC inhibitors on FLIP expression, activation of caspase-8 and drug resistance in NSCLC and normal lung cell line models. Immunohistochemical analysis of cytoplasmic and nuclear FLIP and procaspase-8 protein expression was carried out using a novel digital pathology approach. Both FLIP and procaspase-8 were found to be significantly overexpressed in tumours, and importantly, high cytoplasmic expression of FLIP significantly correlated with shorter overall survival. Treatment with HDAC inhibitors targeting HDAC1-3 downregulated FLIP expression predominantly via post-transcriptional mechanisms, and this resulted in death receptor- and caspase-8-dependent apoptosis in NSCLC cells, but not normal lung cells. In addition, HDAC inhibitors synergized with TRAIL and cisplatin in NSCLC cells in a FLIP- and caspase-8-dependent manner. Thus, FLIP and procaspase-8 are overexpressed in NSCLC, and high cytoplasmic FLIP expression is indicative of poor prognosis. Targeting high FLIP expression using HDAC1-3 selective inhibitors such as entinostat to exploit high procaspase-8 expression in NSCLC has promising therapeutic potential, particularly when used in combination with TRAIL receptor-targeted agents.

Cell Death and Disease (2013) 4, e951; doi:10.1038/cddis.2013.481; published online 5 December 2013

Subject Category: Cancer

Non-small cell lung cancer (NSCLC) is the leading cause of cancer-related mortality in the world. Despite developments in the use of molecular-targeted therapies in stratified patient populations, chemotherapy remains the mainstay of treatment for NSCLC, and drug resistance remains a major challenge that accounts for poor survival outcomes. Novel therapeutic approaches that exploit tumour dependencies on key pathways are urgently needed to improve patient prognosis.

Evasion of apoptosis is a hallmark of cancer and a key cause of therapy. One of the mechanisms by which tumours evade apoptosis is by overexpression of anti-apoptotic proteins such as the caspase-8 inhibitor FLIP, which blocks induction of apoptosis mediated by death receptors such as Fas, DR4 (TRAIL-R1) and DR5 (TRAIL-R2). ${ }^{1}$ FLIP inhibits homo-dimerization, self-processing and activation of procaspase-8 at the death-inducing signalling complexes (DISCs) formed following activation of these receptors. FLIP also blocks apoptosis induced by the DISC-related cytoplasmic caspase-8-activating platforms TNFR1 Complex II and the Ripoptosome. ${ }^{2,3}$

There is emerging pre-clinical and clinical evidence that histone deacetylase inhibitors (HDACi) are promising therapeutic agents for several cancers. However, trials in unselected patient populations in solid tumours have been generally disappointing, highlighting the need for predictive biomarkers to stratify responsive patient populations and identification of rational clinical combinations of these agents with other drugs based on pre-clinical research. ${ }^{4}$ Another class of anti-cancer agent assessed in NSCLC are those targeting DR4 and DR5. Both receptors were found to be highly expressed in NSCLC, ${ }^{5}$ providing support for their clinical assessment in this disease. Overall, clinical trials using recombinant forms of human TRAIL (rhTRAIL) and agonistic antibodies targeting the receptors have been disappointing (reviewed in den Hollander et al. $^{6}$ ). It is important to note however, that these trials were again conducted in unselected patient populations.

\section{Results}

FLIP and procaspase-8 expression in NSCLC. We previously reported that procaspase-8 was overexpressed in $85 \%$ of a small series $(n=20)$ of NSCLC tumours of mixed

\footnotetext{
${ }^{1}$ Drug Resistance Group, Centre for Cancer Research and Cell Biology, Queen's University, Belfast, Northern Ireland, UK; ${ }^{2}$ University of Leicester and Leicester University Hospitals, Leicester, UK; ${ }^{3}$ Department of Cardiothoracic Surgery, St. James's Hospital, Dublin, Ireland and ${ }^{4}$ Department of Pathology, Education and Research Centre, Royal College of Surgeons of Ireland, Beaumont Hospital, Dublin, Ireland

*Corresponding author: D Longley, Centre for Cancer Research and Cell Biology, Queen's University Belfast, 97 Lisburn Road, Belfast, Northern Ireland BT9 7BL, UK. Tel: +44 2890 972762; Fax: +44 2890 972776; E-mail: d.longley@qub.ac.uk

Keywords: non-small cell lung cancer; FLIP; caspase-8; TRAIL; HDAC inhibitor

Abbreviations: cIAP, cellular inhibitor of apoptosis; DISC, death-inducing signalling complex; DR, death receptor; FLIP, FLICE-like inhibitory protein; HDAC, histone deacetylase inhibitor; HR, hazard ratio; NSCLC, non-small cell lung cancer; OS, overall survival; TMA, tissue microarray; TNF, tumour necrosis factor; TRAIL, TNF-related apoptosis inducing ligand; XIAP, X-linked inhibitor of apoptosis

Received 26.7.13; revised 29.10.13; accepted 31.10.13; Edited by M Leverkus
} 
histology. ${ }^{7}$ Moreover, FLIP was overexpressed in all tumours that overexpressed procaspase-8. To assess the levels of procaspase-8 and FLIP expression in a larger NSCLC patient cohort, we generated a tissue microarray (TMA) of 184 samples, comprising three tumour cores and three cores of adjacent normal stroma for each patient. The clinicopathological details of the patient cohort are presented in Table 1.

As described in the materials and methods, FLIP and procaspase-8 expression were assessed by immunohistochemistry and scored using a novel automated image analysis technique, in which expression in the nuclei and cytoplasm were individually scored from digitally captured images of each core (Figures $1 a$ and $b$ and Supplementary Figure 1A). Both proteins could be detected in the cytoplasm and nuclei and were scored separately for each of these cellular compartments. Expression of FLIP and procaspase-8 were significantly higher in the cytoplasm than the nucleus in both normal and tumour samples $(P<0.0001$; Figure 1c). Notably, FLIP and procaspase-8 were expressed at significantly higher levels in tumour tissues than in the stroma $(P<0.0001$; Figure 1c). Squamous cell carcinomas had significantly higher cytoplasmic FLIP expression $(P<0.05)$ than adenocarcinomas,

Table 1 Clinicopathological characteristics of NSCLC patients and their tumours used in this study

\begin{tabular}{|c|c|}
\hline Characteristics & $N(\%)$ \\
\hline $\begin{array}{l}\text { Total } \\
\text { Median age (range) }\end{array}$ & $\begin{array}{c}184(100 \%) \\
66.1(41-86)\end{array}$ \\
\hline $\begin{array}{l}\text { Gender } \\
\text { Male } \\
\text { Female }\end{array}$ & $\begin{array}{r}112(62.7 \%) \\
72(37.3 \%)\end{array}$ \\
\hline $\begin{array}{l}\text { Smoking history } \\
\text { Former/current } \\
\text { Never }\end{array}$ & $\begin{array}{c}171(93.5 \%) \\
13(6.5 \%)\end{array}$ \\
\hline $\begin{array}{l}\text { Histology } \\
\text { Adenocarcinoma } \\
\text { Squamous cell carcinoma } \\
\text { Large cell carcinoma } \\
\text { Pleomorphic } \\
\text { Mixed }\end{array}$ & $\begin{array}{c}83(43.8 \%) \\
83(46.3 \%) \\
4(2.5 \%) \\
7(3.5 \%) \\
7(3.5 \%)\end{array}$ \\
\hline $\begin{array}{l}\text { pTNM stage } \\
\text { II } \\
\text { III }\end{array}$ & $\begin{array}{l}99(54.2 \%) \\
43(23.9 \%) \\
42(21.9 \%)\end{array}$ \\
\hline $\begin{array}{l}\text { Tumour size } \\
\qquad 2 \mathrm{~cm} \\
>2-3 \mathrm{~cm} \\
>3-5 \mathrm{~cm} \\
>5-7 \mathrm{~cm} \\
>7 \mathrm{~cm}\end{array}$ & $\begin{array}{l}20(11.4 \%) \\
31(16.9 \%) \\
89(46.3 \%) \\
24(14.4 \%) \\
20(10.9 \%)\end{array}$ \\
\hline $\begin{array}{l}\text { Type of resection } \\
\text { Lobectomy } \\
\text { Bilobectomy } \\
\text { Pneumonectomy }\end{array}$ & $\begin{array}{r}135(72.1 \%) \\
17(10.4 \%) \\
32(17.4 \%)\end{array}$ \\
\hline $\begin{array}{l}\text { Tumour grade } \\
\text { G1 } \\
\text { G2 } \\
\text { G3 }\end{array}$ & $\begin{array}{c}11(6.5 \%) \\
110(58.7 \%) \\
63(34.8 \%)\end{array}$ \\
\hline
\end{tabular}

although overall, the patterns of FLIP and procaspase-8 expression in squamous and adenocarcinomas were very similar (Supplementary Figure 1B). Also in agreement with our previous study, there were significant correlations between FLIP and procaspase-8 expression in both the nuclear and cytoplasmic compartments (Figure 1d). There were no correlations between procaspase-8 and FLIP expression levels and gender, smoking history or tumour grade (data not shown).

Comparison of Kaplan-Meier curves for low (less than the median) versus high (greater than the median) expression revealed no significant correlation within 5 years of follow-up for nuclear FLIP, nuclear procaspase-8 and cytoplasmic procaspase-8, however there was a significant correlation $(P=0.032)$ for high cytoplasmic FLIP expression being associated with shorter OS (Figure 1e; HR=1.59). The correlation of high cytoplasmic FLIP with poor prognosis was also apparent in both adenocarcinoma and squamous histological sub-types and was more marked in the squamous tumours, although these correlations failed to reach significance in these smaller sub-groups (adenocarcinoma: $P=0.15, \quad H R=1.54$; squamous: $P=0.07, \quad H R=1.98$; Supplementary Figure 2).

HDAC inhibitors induce FLIP downregulation and cell death in NSCLC cells. The high frequency of procaspase-8 and FLIP overexpression and the correlation of high cytoplasmic FLIP expression with poor prognosis suggested that a significant proportion of NSCLCs may be vulnerable to procaspase-8-mediated apoptosis if the inhibitory effects of FLIP overexpresion could be therapeutically overcome. Western blot analyses demonstrated that knockdown of both major FLIP splice forms (FLIPS and FLIP $)$ in the H460 and A549 NSCLC cell line models was sufficient to induce PARP cleavage, indicative of apoptosis (Figure 2a), showing that these NSCLC cell lines are dependent on FLIP to maintain viability. Vorinostat and panobinostat are pan-HDAC inhibitors that have been identified by us and others as modulators of FLIP expression in several pre-clinical cancer models. ${ }^{8-10}$ Treatment with either of these HDAC inhibitors for $24 \mathrm{~h}$ resulted in downregulation of FLIP protein expression in NSCLC cells at clinically achievable doses (Figure $2 b$ and Supplementary Figure 3A). Moreover, significant downregulation of both major FLIP splice forms was observable $6 \mathrm{~h}$ after vorinostat treatment. FLIP downregulation in response to both pan-HDAC inhibitors correlated with caspase-3 activation and PARP cleavage at $24 \mathrm{~h}$ (Figure $2 \mathrm{~b}$ and Supplementary Figure $3 A-C)$. The only other major antiapoptotic protein downregulated in response to HDAC inhibitors was XIAP, which was significantly downregulated after $24 \mathrm{~h}$ in both cell lines (Supplementary Figure 3B); interestingly, vorinostat synergized with the XIAP antagonist Birinapant in $\mathrm{H} 460$ and A549 cells (Supplementary Figure 4D).

Vorinostat potently suppressed FLIP expression for $72 \mathrm{~h}$ and decreased procaspase-8 expression (an indication of processing and activation) was observed (Figure 2c). In a normal lung fibroblast cell line, 34LU, FLIPS expression was significantly lower (Figure 2d) and FLIP was only downregulated at the highest concentrations of HDAC inhibitor 

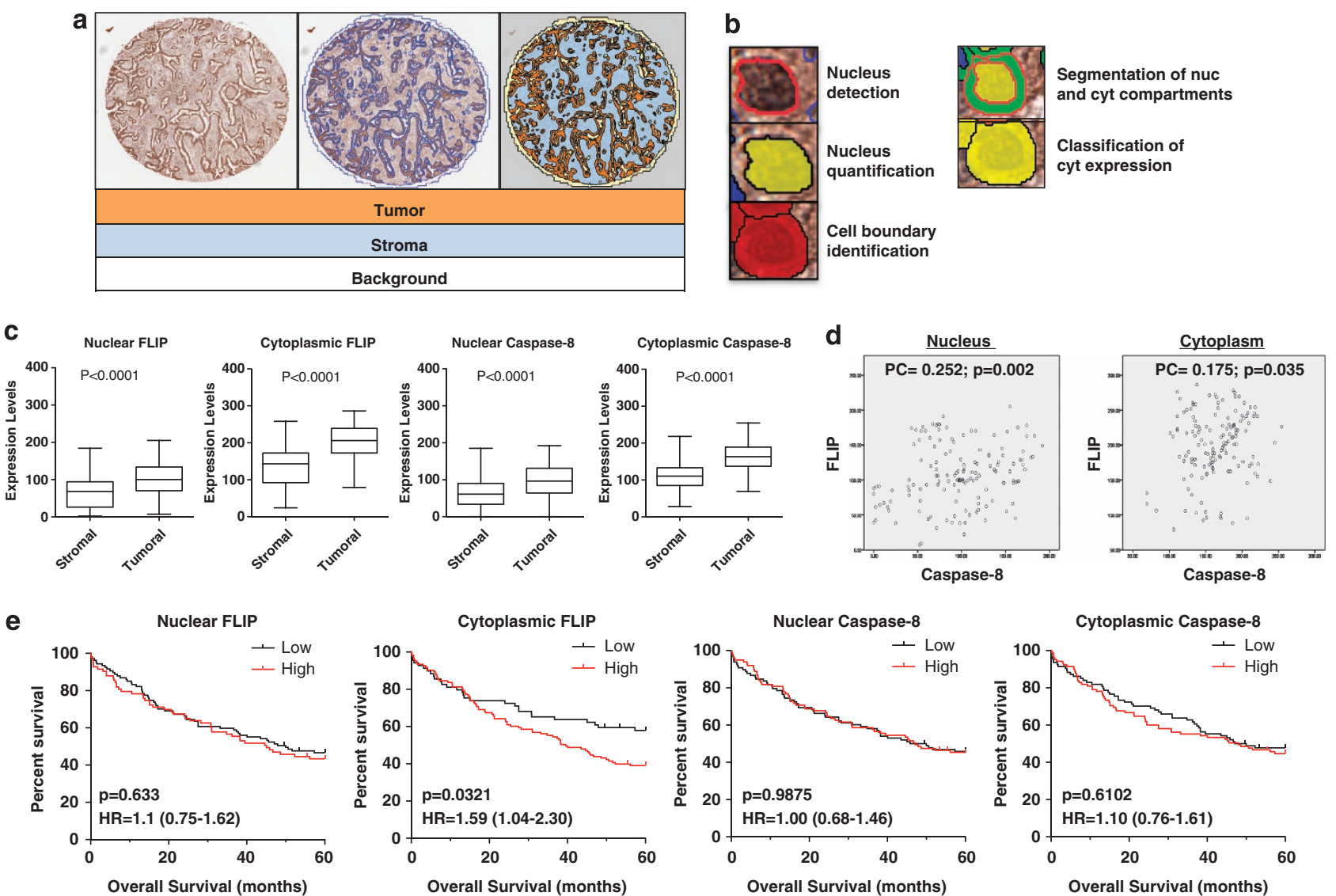

Figure 1 FLIP and procaspase-8 expression in tumour samples; clinical correlates. (a) Automated tumour identification ('region recognition module') of lung adenocarcinoma tissue stained with FLIP antibody. (b) The 'cellular detection module': nucleus detection, nucleus quantification, cell boundary identification, segmentation of nucleus and cytosolic compartments and classification of cytosolic expression. (c) Absolute expression of FLIP and procaspase-8 in nuclei and cytoplasm across all patients. Significance determined by Mann-Whitney non-parametric, two-tailed $t$-test. (d) Correlations between tumoral expression of FLIP and procaspase-8 in nuclei (left) and cytoplasm (right), as determined using Pearson's correlation. (e) Correlations of disease-free survival with the level of nuclear and cytoplasmic FLIP or procaspase-8 expression in tumour tissue across all patients. Statistical significance of Kaplan-Meier curves was determined by the log-rank (Mantel-Cox) test

used, and then only after $24 \mathrm{~h}$ (Figure $2 \mathrm{e}$ and Supplementary Figure 3D). Compared with the NSCLC models, vorinostat and panobinostat induced significantly less apoptosis in 34LU cells as determined by PARP cleavage (Figure $2 \mathrm{e}$ and Supplementary Figure 3D) and flow cytometry (Figure 2f).

Vorinostat downregulated FLIP mRNA levels by $\sim 50 \% 6 \mathrm{~h}$ post treatment in each cell line (Supplementary Figure $4 A$ ). In H460 cells, FLIP mRNA expression recovered to control levels by $24 \mathrm{~h}$; whereas in A549 cells, FLIP mRNA expression remained downregulated. However, in both cell lines, the downregulation of FLIP mRNA expression was insufficient to account for the downregulation of FLIP protein expression, particularly at later timepoints, suggesting that vorinostat predominantly suppresses FLIP protein expression at a posttranscriptional level in these models. FLIP is a known caspase-8 substrate; therefore we investigated whether FLIP downregulation post-HDAC inhibitor treatment was due to activation of caspases. Notably, FLIP downregulation in response to vorinostat was unaffected by co-treatment with the pan-caspase inhibitor z-VAD-fmk (Supplementary Figure 4B). However, HDAC inhibitor-induced downregulation of FLIP was blocked in cells pre-treated with the proteasome inhibitor MG132 (Supplementary Figure 4C), indicating that the ubiquitin-proteasome pathway is involved in HDAC inhibitor-mediated FLIP downregulation in NSCLC.

Vorinostat-induced cell death is dependent on the extrinsic apoptotic pathway. In both $\mathrm{H} 460$ and A549 cells, downregulation of procaspase-8 rescued the cell death phenotype induced by vorinostat (Figure 3a), demonstrating that the apoptosis induced by this agent is dependent on activation of caspase-8. Notably, vorinostat-resistant 34LU fibroblasts expressed significantly lower levels of procaspase-8 than the NSCLC cell lines (Figure 2d). Treatment of FLIP-overexpressing cells with vorinostat resulted in significantly less apoptosis compared with the empty vector (EV) control line (Figure 3b). Collectively, these data show that vorinostat-induced cell death is critically dependent on FLIP downregulation and caspase-8 activation. Furthermore, we found that vorinostat-induced apoptosis after 24 and $48 \mathrm{~h}$ was significantly attenuated in $\mathrm{H} 460$ and A549 cells in which either DR4 or DR5 (but not Fas) was downregulated 
a

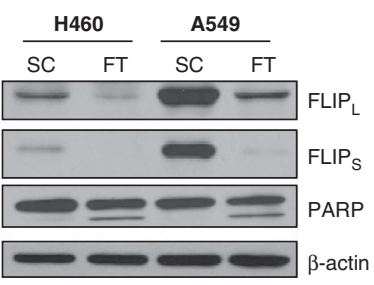

C

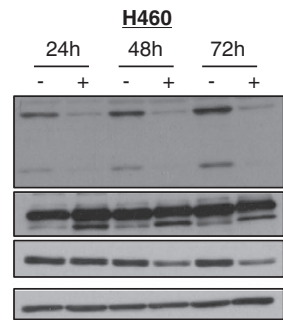

e

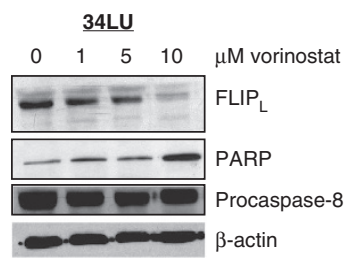

b
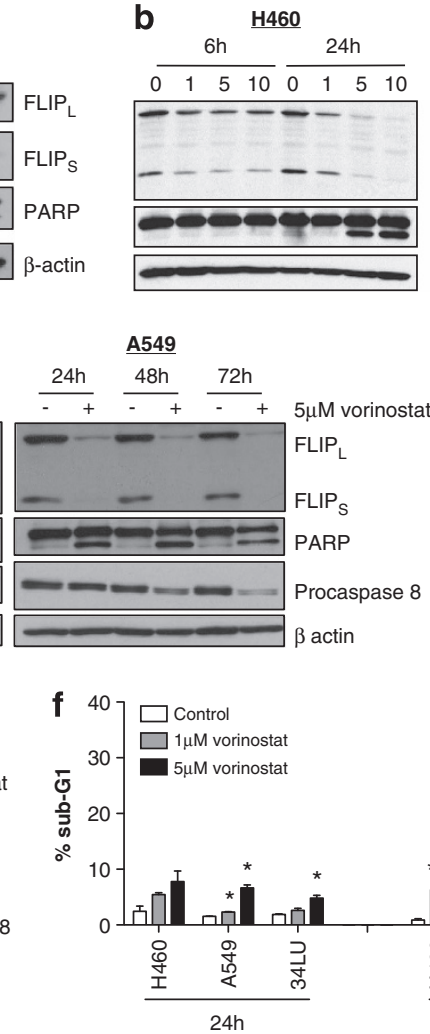
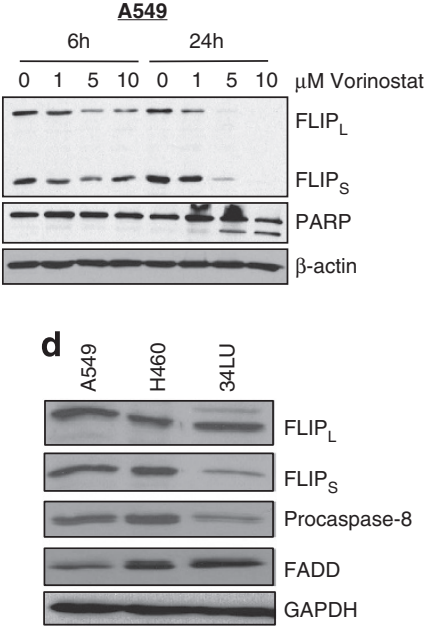

d

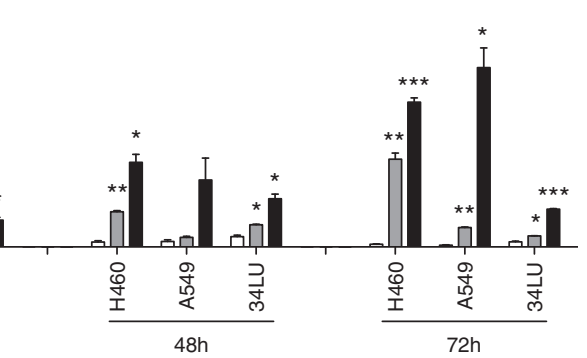

Figure 2 HDACi induce FLIP downregulation and apoptosis in NSCLC cell lines. (a) Western blot analysis of FLIP expression and PARP cleavage in H460 and A549 cells transfected with FLIP-targeted siRNA (FT) for $24 \mathrm{~h}$. (b) Western blot analysis of FLIP expression and PARP cleavage in H460 and A549 cells treated with the indicated doses of vorinostat for 6 or $24 \mathrm{~h}$. (c) Western blot analysis of FLIP and procaspase-8 expression and PARP cleavage in H460 and A549 cells treated with $5 \mu \mathrm{M}$ vorinostat for the indicated timepoints. (d) Western blot analysis of basal FLIP, procaspase-8 and FADD expression in H460 and A549 NSCLC cells and 34LU normal lung fibroblasts. (e) Western blot analysis of FLIP and procaspase-8 expression and PARP cleavage in 34LU cells treated with vorinostat for $24 \mathrm{~h}$. (f) Flow cytometric analysis of apoptosis (sub-G1 population) in H460, A549 and 34LU cells treated with vorinostat for the indicated times. Significant differences were determined by Student's $t$-test: ${ }^{*} P<0.05 ;{ }^{* \star} P<0.01 ;{ }^{* * \star} P<0.001$

(Figure $3 \mathrm{c}$ and data not shown), providing further evidence for the involvement of the extrinsic apoptotic pathway in mediating vorinostat-induced apoptosis.

Loss of expression of BAK has been observed in $34-47 \%$ of surgically resected NSCLC tumours, and loss of BAX expression in $42-59 \% .{ }^{11}$ As these proteins are potent apoptosis activators, this downregulation could potentially mediate profound chemo-resistance. We assessed whether BAX/BAK-deficient tumours were resistant to treatment with HDAC inhibitors by analysing two $\mathrm{H} 460$ daughter cell lines stably expressing shRNAs targeting BAX and BAK (Figure 3d). Notably, the levels of apoptosis induced by vorinostat in the BAX/BAK-deficient cells were comparable to those in a control cell line expressing non-targeting shRNA (Figures $3 e$ and f).

\section{Vorinostat potentiates the effect of cisplatin in NSCLC} cells. Cisplatin is a DNA-damaging agent used as first-line treatment for patients with stage IV NSCLC, although only $\sim 20 \%$ of patients respond. ${ }^{12,13}$ Co-treatment with vorinostat and cisplatin increased PARP cleavage and processing of procaspase- 3 into its active form compared with treatment with each agent individually (Figure 4a). In addition, vorinostat and cisplatin co-treatment dramatically reduced long-term clonogenic survival compared with either of the drugs alone in both cell lines (Figure $4 \mathrm{~b}$ and Supplementary Figure $5 \mathrm{C}$ ), and short-term cell viability assays clearly demonstrated strong synergy between the two drugs, with combination indices (Cls) for nearly all dose combinations $<1$, with many of them falling between 0.3 and 0.7 (Figure 4c). Mechanistically, FLIP overexpression reduced PARP cleavage and processing of procaspase- 8 to its $p 18$ form in response to combined cisplatin/vorinostat co-treatment (Figure 4d), whereas RNAi-mediated procaspase-8 depletion blocked the additional apoptosis induced when vorinostat was co-administered with cisplatin (Figure 4e). Thus, the synergy between cisplatin and vorinostat is dependent on FLIP downregulation and caspase-8 activation; although it should be noted that neither FLIP overexpression nor procaspase-8 depletion inhibited the apoptosis induced by cisplatin alone (Figures $4 \mathrm{~d}$ and e).

Vorinostat synergizes with TRAIL in NSCLC cells. As FLIP is a potent inhibitor of TRAIL-mediated apoptosis, we postulated that pre-treating cells with HDAC inhibitors to downregulate FLIP would overcome resistance to TRAIL in NSCLC. Indeed, co-treatment of $\mathrm{H} 460$ and A549 cells with vorinostat and TRAIL resulted in significant apoptosis induction as assessed by PARP cleavage and caspase-3 processing (Figure $5 \mathrm{a}$ ). This combination also synergistically 

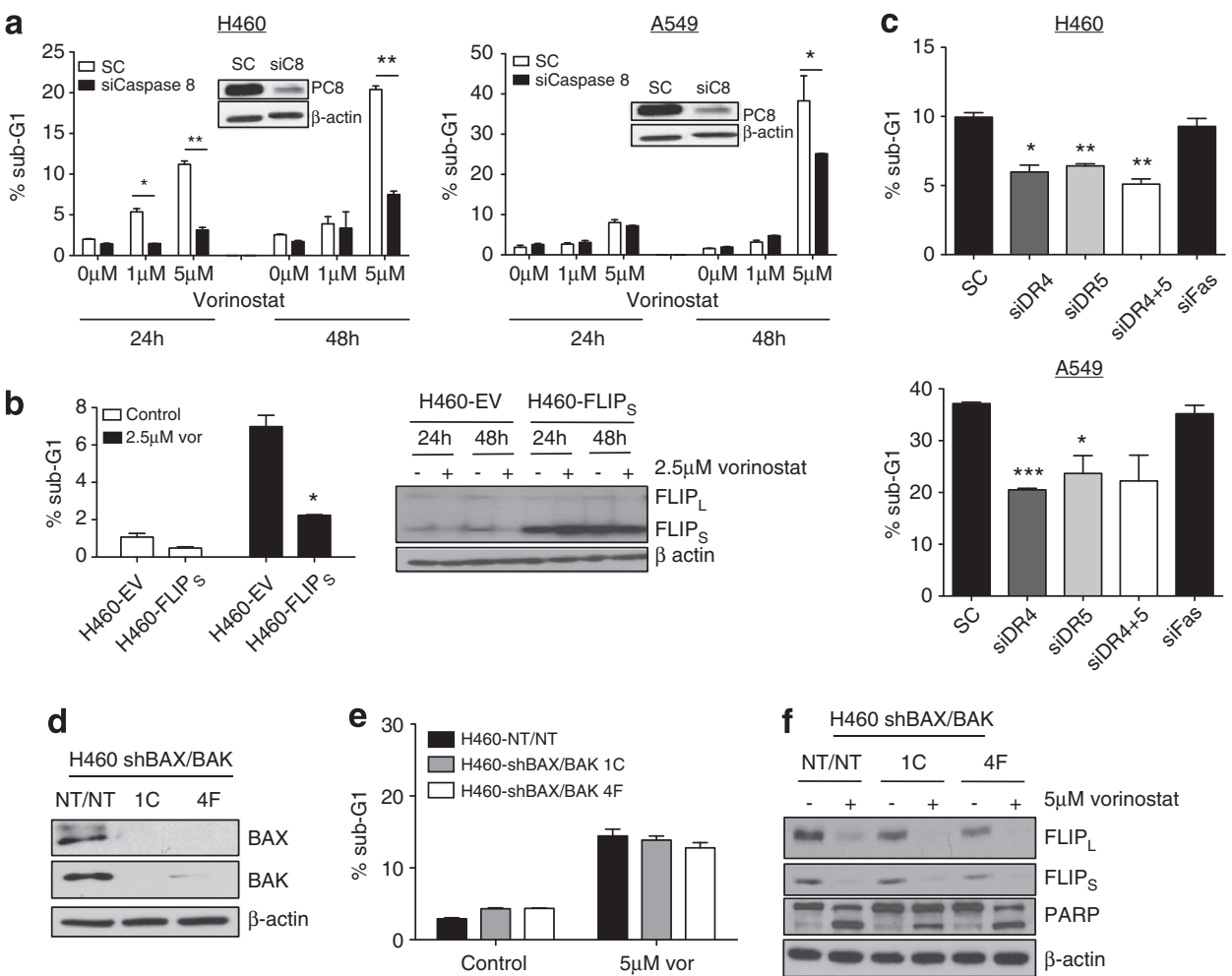

Figure 3 HDACi-induced apoptosis of NSCLC cells is dependent on the extrinsic apoptotic pathway. (a) Flow cytometric analysis of apoptosis (sub-G1 population) in H460 and A549 cells treated with 1 or $5 \mu \mathrm{M}$ vorinostat for 24 or $48 \mathrm{~h}$. Cells were transfected with $20 \mathrm{nM}$ procaspase-8-targeted (siCaspase-8) or control (SC) siRNA for $48 \mathrm{~h}$ before vorinostat treatment. Efficiency of procaspase-8 depletion is shown by immunoblot. Significant differences were determined using Student's t-test. (b) Flow cytometric analysis of apoptosis (sub-G1 population) in control (EV) and FLIPs overexpressing $\mathrm{H} 460$ cells treated with $2.5 \mu \mathrm{M}$ vorinostat for $48 \mathrm{~h}$. FLIP overexpression was determined by western blotting, and significance was determined using Student's t-test. (c) Death receptors DR4, DR5 or Fas were silenced for $48 \mathrm{~h}$ with $20 \mathrm{nM}$ specific siRNA, following which the cells were treated with $5 \mu \mathrm{M}$ vorinostat for a further $24 \mathrm{~h}$. Sub-G1 populations were analysed by flow cytometry. Significance was determined by Student's $t$-test. (d) Western blot analysis of BAX and BAK in two H460 cell lines stably expressing shRNAs targeting BAX and BAK (shBAX/BAK) or non-targeting (shNT/NT) shRNAs. (e) Sub-G1 analysis of apoptosis in shBAX/BAK cells treated with $5 \mu \mathrm{M}$ vorinostat for $48 \mathrm{~h}$. (f) Western blot analysis of FLIP expression and PARP cleavage in shBAX/BAK cells treated for $48 \mathrm{~h}$ with $5 \mu \mathrm{M}$ vorinostat. Significant differences were determined by Student's $t$-test: ${ }^{*} P<0.05 ;{ }^{* \star} P<0.01 ;{ }^{* * \star} P<0.001$

decreased long-term clonogenic survival (Figure $5 b$ and Supplementary Figure $5 \mathrm{C}$ ) and short-term cell viability as determined by the $\mathrm{Cl}$ method (Figure 5c). Furthermore, $\mathrm{H} 460$ cells that have acquired resistance to cisplatin ${ }^{14}$ still undergo apoptosis in response to FLIP downregulation and vorinostat (Supplementary Figure $5 \mathrm{~A}-\mathrm{C}$ ). Moreover, these cisplatin-resistant cells are as sensitive as the parental cells to combined treatment with vorinostat and TRAIL; however vorinostat did not reverse their acquired resistance to cisplatin (Supplementary Figure 6D). Mechanistically, overexpression of FLIP protected cells from apoptosis induced by TRAIL alone, vorinostat alone and the combination as assessed by PARP cleavage and processing of procaspase-8 to its p18 subunit (Figure 5d). Similarly, RNAi-mediated procaspase-8 depletion significantly protected cells from apoptosis induced by TRAIL and vorinostat (Figure 5e).

Inhibition of HDACs 1,2 and 3 is required for FLIP downregulation. HDACs 1, 2 and 3 were found to be more highly expressed in the NSCLC cell lines than in the normal fibroblasts, whereas HDAC6 (detected as two bands) was higher in the fibroblasts (Figure 6a). While vorinostat and panobinostat inhibit a broad spectrum of HDACs, ${ }^{15}$ HDAC inhibitors with greater specificity have been developed, such as entinostat (MS-275), which inhibits HDACs 1, 2 and 3, but not HDAC6, and ACY-775, which is highly selective for HDAC 6 but has no activity against HDACs $1-3 .^{16,17}$ Notably, entinostat, but not ACY-775, induced significant downregulation of FLIP protein expression in $\mathrm{H} 460$ and A549 cells (Figure 6b), suggesting that HDACs 1-3, but not HDAC6, are important for regulating FLIP expression in NSCLC. Entinostat also downregulated FLIP expression in 34LU cells (Figure 6c). Co-treatment with entinostat enhanced cisplatin-induced apoptosis in A549 cells (albeit to a lesser extent than vorinostat), but not $\mathrm{H} 460$ cells as determined by PARP cleavage and caspase-3 activation (Figure 6c) and flow cytometry (Figure 6d). However, similar to vorinostat, entinostat synergistically enhanced TRAIL-induced apoptosis in both NSCLC cell lines (Figures $6 c$ and d). Of note, entinostat was significantly less toxic than vorinostat to 34LU cells, either as a single agent or in combination with TRAIL (Figures $6 \mathrm{c}$ and $\mathrm{d}$ ); although similar to vorinostat, it enhanced cisplatin-induced apoptosis in the normal lung cells (Figures $6 c$ and $d$ ). Like vorinostat, the effects of entinostat on TRAIL- and cisplatin-induced apoptosis were caspase-8-dependent (Figure 6e), consistent with a FLIP-dependent mode of cell death induction. 


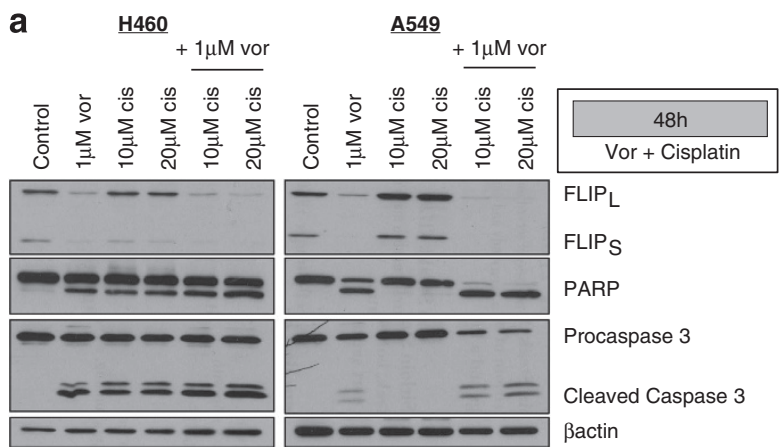

d

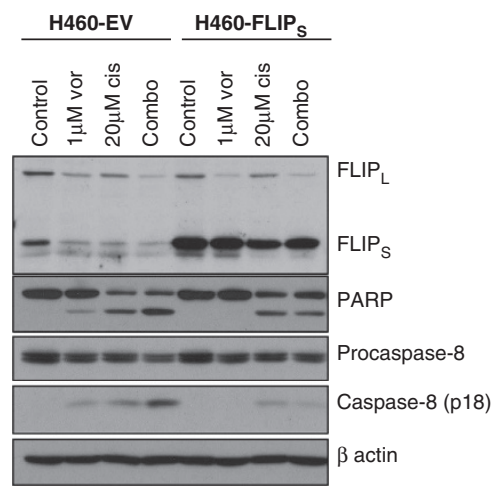

b
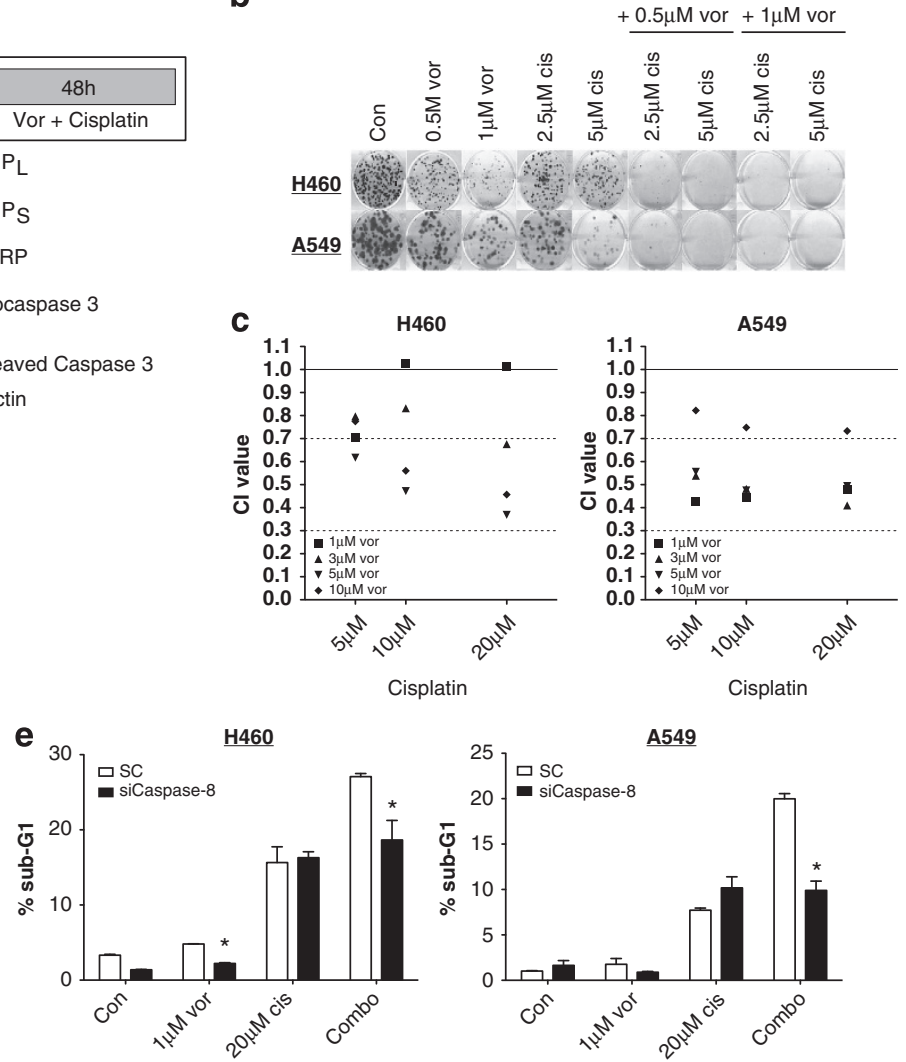

Figure 4 HDACi synergize with cisplatin. (a) FLIP expression, cleavage of PARP and processing of procaspase-3 in H460 and A549 cells were determined by western blotting. Cells were co-treated with the indicated concentrations of vorinostat and cisplatin for $48 \mathrm{~h}$. (b) Colony formation was assessed 2 weeks following treatment of $\mathrm{H} 460$ and A549 cells with vorinostat alone, cisplatin alone or co-treatment with both agents at the concentrations indicated. After $48 \mathrm{~h}$ of treatment, the media was removed, cells washed in PBS and fresh media added. (c) Viability of $\mathrm{H} 460$ and A549 cells was assessed $48 \mathrm{~h}$ after co-treatment with cisplatin and vorinostat. The cell viability data was used to assess synergy using the $\mathrm{Cl}$ method. (d) Western blot analysis of FLIP expression, procaspase-8 processing and PARP cleavage in control (EV) and FLIPS overexpressing H460 cells treated as indicated for 48 h. (e) Sub-G1 analysis of apoptosis in H460 and A549 cells treated with vorinostat alone, cisplatin alone or co-treated for $48 \mathrm{~h}$ with both agents (combo). Cells were transfected with $20 \mathrm{nM}$ procaspase-8-targeted siRNA (siCaspase-8) or control siRNA (SC) for $48 \mathrm{~h}$ before cisplatin/vorinostat treatment. Significance was determined using Student's $t$-test: ${ }^{*} P<0.05$

\section{Discussion}

NSCLC is particularly resistant to chemotherapy. Mutations or alterations in expression of proteins regulating apoptosis often arise during carcinogenesis or during treatment; this can be a major cause of clinical drug resistance, therefore therapies that specifically target these proteins are being actively sought. ${ }^{18-21}$ One of these anti-apoptotic proteins is FLIP, which is overexpressed in several cancer types and has been associated with drug resistance and poor prognosis, ${ }^{1}$ but for which there are currently no direct inhibitors.

In this study, we used a novel automated scoring technique to assess nuclear and cytoplasmic expression of FLIP and procaspase-8 in tumour and adjacent stroma tissue from a cohort of 184 stage I-III patients with mixed histology NSCLC. Unlike small cell lung cancer in which procaspase-8 expression is frequently downregulated or deleted, ${ }^{22}$ NSCLC tumours expressed significantly higher levels of procaspase8 compared with surrounding stromal tissue. FLIP was also frequently overexpressed, and we observed positive correlations between the levels of FLIP and procaspase-8 expression within the tumour tissues. Notably, we found a significant correlation between high cytoplasmic FLIP expression and poor OS that was not apparent for nuclear FLIP expression, consistent with the primary function of FLIP as a caspase-8 inhibitor being cytoplasmic. ${ }^{1}$ Further analyses in an expanded patient cohort are now required to assess whether high cytoplasmic FLIP expression as assessed using this automated scoring approach has clinical utility as a biomarker of poor prognosis in NSCLC.

We have previously shown that RNAi- and antisensemediated FLIP downregulation induces apoptosis in NSCLC cell lines in vitro and in vivo ${ }^{7,23}$ and that the sensitivity of NSCLC cells to FLIP downregulation is dependent on their elevated expression of procapase- $8 .{ }^{7}$ Thus, FLIP represents an attractive therapeutic target in this disease. There have been a number of in vitro studies in a range of malignancies showing FLIP downregulation in response to HDAC inhibitors. ${ }^{8,10,24-27}$ Although FLIP downregulation following treatment with pan-HDAC inhibitors has been documented, it is not known which particular HDACs are responsible for mediating this effect in different tumour types. Our results show that inhibition of HDAC1, 2 and/or 3, but not HDAC6, is necessary for efficient FLIP downregulation in NSCLC. This is interesting given the predominantly (but not exclusively) nuclear expression of these HDACs. ${ }^{28}$ 

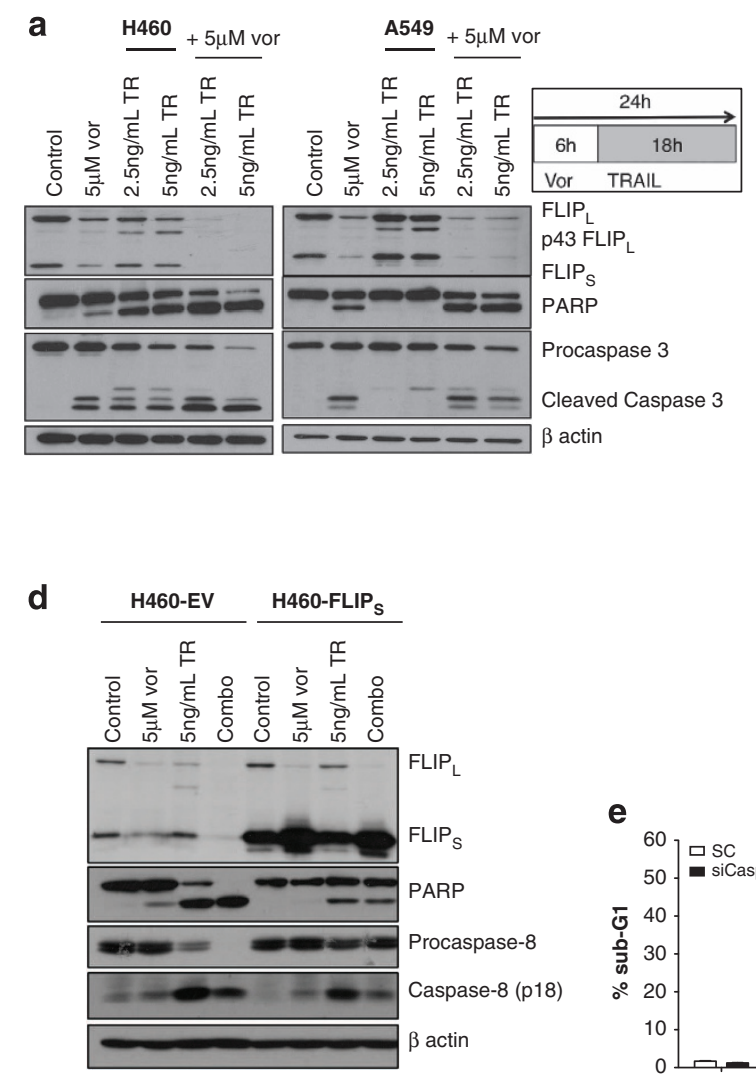

b

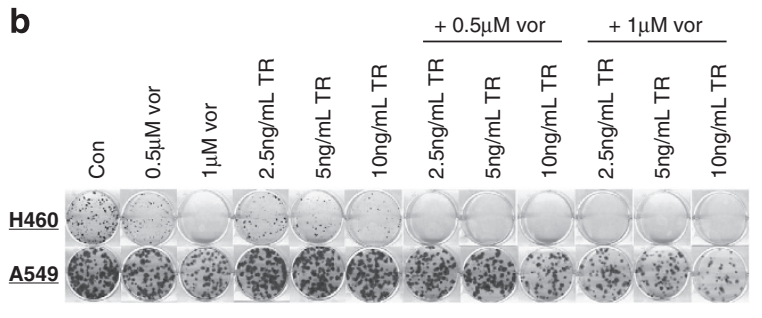

c
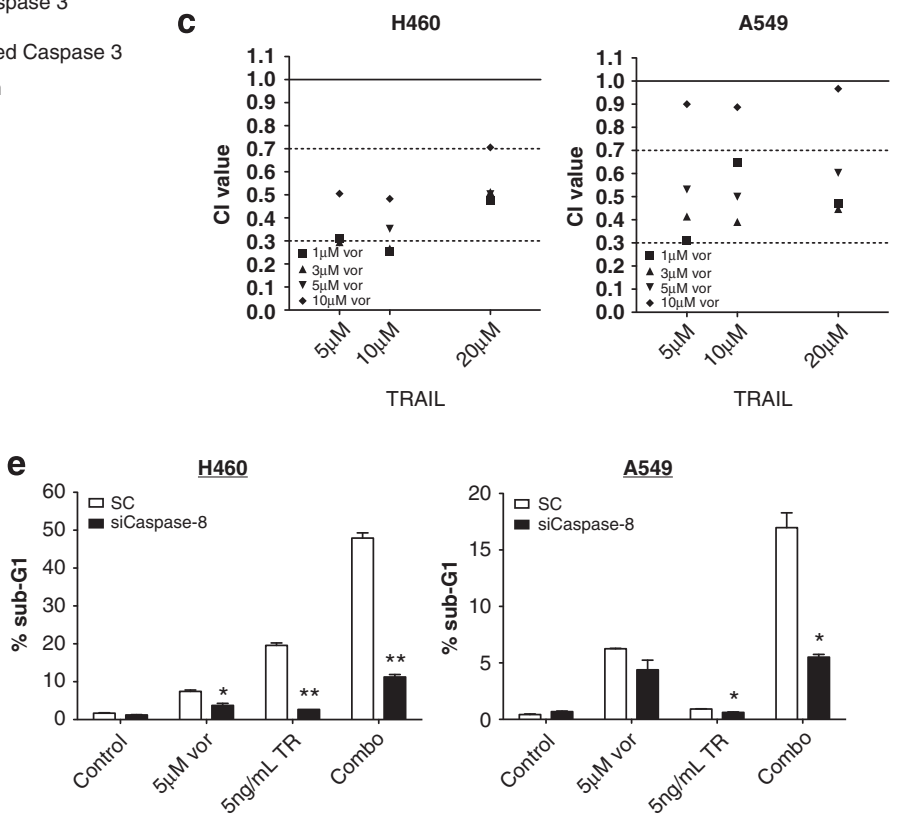

Figure 5 HDACi synergize with TRAIL. (a) FLIP expression, cleavage of PARP and processing of procaspase-3 in H460 and A549 cells were determined by western blotting. Cells were pre-treated with $5 \mu \mathrm{M}$ vorinostat for $6 \mathrm{~h}$ prior to treatment with TRAIL for a further $18 \mathrm{~h}$. (b) Colony formation was assessed 2 weeks following treatment of $\mathrm{H} 460$ and A549 cells with vorinostat alone, TRAIL alone or co-treatment with both agents at the concentrations indicated. After $24 \mathrm{~h}$ of treatment, the media was removed, cells washed in PBS, and fresh media added. (c) Viability of $\mathrm{H} 460$ and A549 cells was assessed $72 \mathrm{~h}$ after co-treatment with TRAlL and vorinostat. The cell viability data was used to assess synergy using the $\mathrm{Cl}$ method. (d) Western blot analysis of FLIP expression, procaspase-8 processing and PARP cleavage in control (EV) and FLIPS overexpressing $\mathrm{H} 460$ cells treated as indicated for $24 \mathrm{~h}$. (e) Sub-G1 analysis of apoptosis in H460 and A549 cells treated with vorinostat alone, TRAlL alone or co-treated with both agents (combo). Cells were transfected with $20 \mathrm{nM}$ procaspase-8-targeted siRNA (siCaspase-8) or control siRNA (SC) for $48 \mathrm{~h}$ before TRAlL/vorinostat treatment. Significance was determined using Student's $t$-test: ${ }^{*} P<0.05 ;{ }^{* *} P<0.01$

HDAC inhibitors are a novel class of agents with a variety of chemical structures that are thought to exert their anticancer effects by epigenetically altering gene expression. ${ }^{29}$ However, it is becoming increasingly apparent that effects on the epigenome may not be the only (or indeed primary) anticancer mechanism of action of HDAC inhibitors, which in turn has implications for how these drugs should be rationally combined with other agents to maximize their therapeutic potential. ${ }^{30,31}$ There are many HDAC inhibitors at various stages of clinical development and, to date, vorinostat and Istodax/romidepsin have been approved for use in cutaneous T-cell lymphoma. ${ }^{32-35}$ The benzamide entinostat (MS-275/ SNDX-275) is an HDAC1/2/3-selective inhibitor that has been in numerous clinical trials, including several in NSCLC most often combined with the demethylatng agent azacitidine. ${ }^{36}$ Entinostat is generally well tolerated and has a significantly longer plasma half-life in man than the hydroxamic acid HDAC inhibitors such as vorinostat and panobinostat. ${ }^{36}$

Cisplatin, in combination with pemetrexed, is currently the first-line standard-of-care chemotherapy for patients with NSCLC. ${ }^{12,13}$ Notably, co-treatment with vorinostat, synergistically enhanced cisplatin-induced apoptosis in NSCLC cells, and this increased apoptosis was attenuated by FLIP overexpression or caspase-8 silencing. There is already clinical evidence for combining platinum drugs with HDAC inhibitors: a phase II trial of carboplatin/ paclitaxel with or without vorinostat in NSCLC reported significantly improved response rates and a trend towards improved survival for the vorinostat arm. ${ }^{37}$ This suggests that there is a cohort of NSCLC patients who will benefit from addition of an HDAC inhibitor to platinum-based chemotherapy.

TRAIL and agonistic TRAIL receptor antibodies are currently being assessed for use as anti-cancer agents. ${ }^{38}$ Although well tolerated, the results of clinical trials in solid tumours have been disappointing so far; however, it is important to note that all trials to date have been conducted in unselected patient populations due to lack of predictive biomarkers for this class of agent. ${ }^{6}$ We found that co-treatment with vorinostat or entinostat sensitized NSCLC cells to TRAIL in a FLIP- and caspase-8dependent manner. This result is in agreement with other studies; for example, Frew et al. ${ }^{24}$ reported that vorinostat 
a

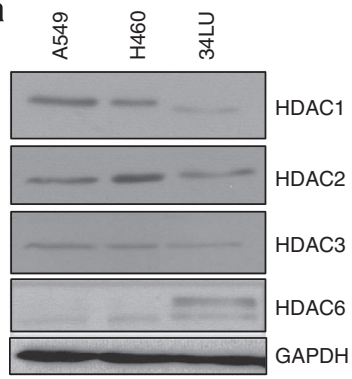

b

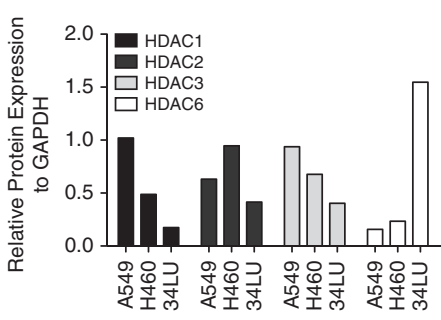

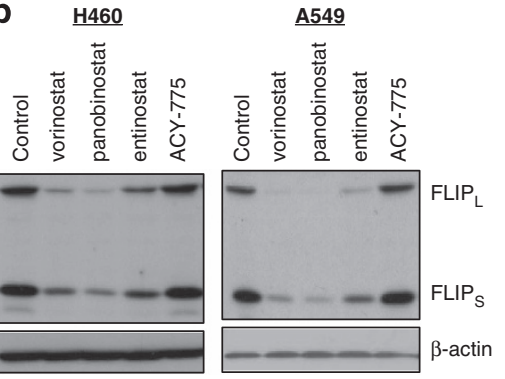

c

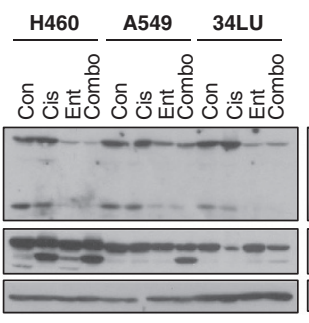

e

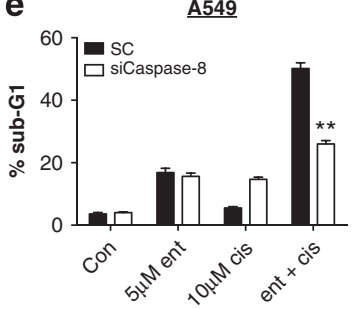

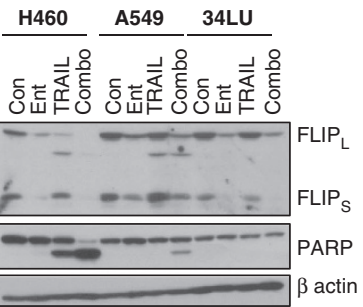

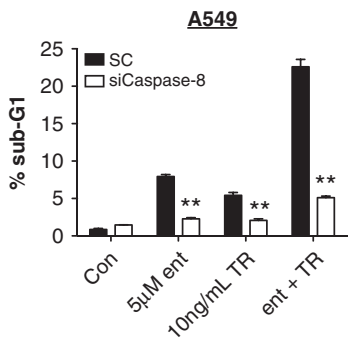

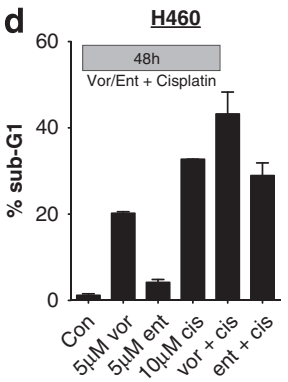
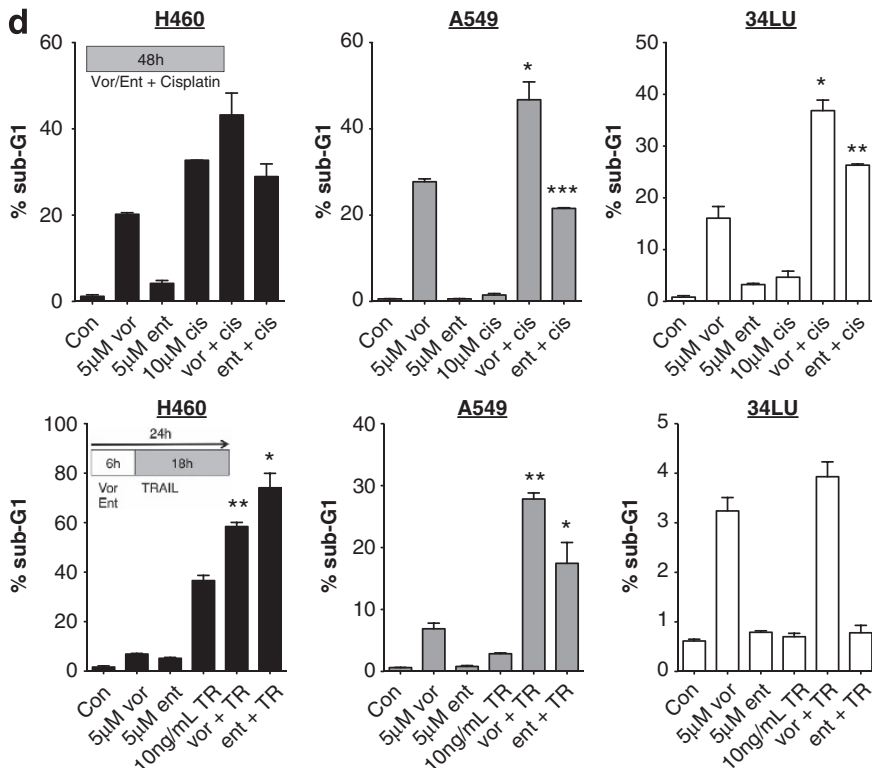

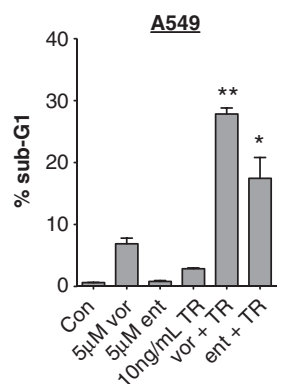

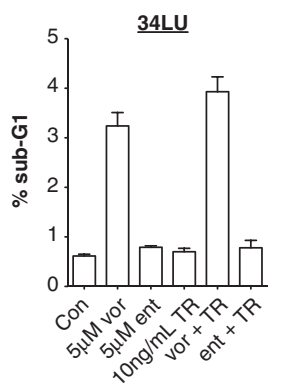

Figure 6 The HDAC1-3 inhibitor entinostat phenocopies the pan-HDAC inhibitor vorinostat. (a) Basal expression of HDAC1, 2, 3 and 6 in A549, H460 and 34LU cell lines was assessed by western blotting and quantified by densitometry relative to GAPDH. (b) Effect of different HDAC inhibitors on FLIP expression was determined by western blotting. The cells were treated for $24 \mathrm{~h}$ with $5 \mu \mathrm{M}$ vorinostat, $50 \mathrm{nM}$ panobinostat (pan-HDAC inhibitors), $5 \mu \mathrm{M}$ entinostat (HDAC1-3-selective inhibitor) or $5 \mu \mathrm{M}$ ACY-775 (HDAC6-selective inhibitor). (c) Western blot analysis of FLIP expression and PARP cleavage in H460, A549 and 34LU cell lines treated with combinations of entinostat and cisplatin for $48 \mathrm{~h}$ or entinostat and TRAIL for $24 \mathrm{~h}$. (d) H460, A549 and 34LU cells were treated with entinostat or vorinostat in combination with cisplatin for $48 \mathrm{~h}$ or TRAIL for $24 \mathrm{~h}$ as indicated. Apoptosis was determined by sub-G1 analysis. Significance was assessed by two-way ANOVA. (e) A549 cells were either co-treated with entinostat and cisplatin for $48 \mathrm{~h}$, or pre-treated with entinostat for $6 \mathrm{~h}$, followed by TRAIL addition for a further $18 \mathrm{~h}$. Cells were transfected with $20 \mathrm{nM}$ procaspase-8-targeted siRNA (siCaspase-8) or control siRNA (SC) for $48 \mathrm{~h}$ before cisplatin/entinostat and TRAIL/entinostat treatment. Significance was determined using Student's $t$-test: ${ }^{*} P<0.05 ;{ }^{* *} P<0.01 ;{ }^{* * *} P<0.001$

acted synergistically with MDS-1 (an anti-TRAIL-R agonistic monoclonal antibody) in a panel of cancer cell lines and caused tumour regression in a mouse breast cancer model. We have previously demonstrated increased TRAIL sensitivity following treatment with vorinostat in mesothelioma and colorectal cancer models, which was also FLIP- and caspase-8-dependent. ${ }^{8,10}$ We postulate that resistance to TRAIL agonists in NSCLC is mediated by high levels of cytoplasmic FLIP expression and may therefore be overcome by co-treatment with HDAC inhibitors. Notably, unlike addition of cisplatin, the addition of TRAIL to vorinostat did not further increase apoptosis in normal lung fibroblasts, suggesting that there may be a wider therapeutic window for this combination. In this regard, entinostat was as effective as vorinostat at enhancing TRAIL-induced apoptosis in the NSCLC models, but this combination had no effect at all on the normal lung cells. Moreover, entinostat alone was less toxic to the normal cells than vorinostat, suggesting that this more selective HDAC inhibitor would be the better tolerated, in agreement with clinical observations. ${ }^{36}$
To maximize the therapeutic impact of HDAC inhibitors in NSCLC, predictive biomarkers for their targeted use in selected patient populations are urgently needed. TRAIL receptor-targeted therapeutics also currently lack predictive biomarkers of response. Our data suggest that tumours with high cytoplasmic FLIP and/or procaspase-8 expression may be particularly responsive to HDAC inhibitor-based combination therapies, in particular combinations with TRAIL receptortargeted agents. To begin to assess this, automated scoring techniques of the type described herein could be used to retrospectively assess whether cytoplasmic FLIP and/or procaspase-8 expression in tumours correlates with sensitivity or resistance in clinical trials of HDAC inhibitors ${ }^{37}$ and TRAIL-targeted therapeutics (reviewed in den Hollander et al. ${ }^{6}$ ) in NSCLC.

\section{Materials and Methods}

Patients. This is a retrospective study of patients who underwent curative-intent surgical resection of a primary tumour at St. James's Hospital, Dublin between February 2001 and February 2005. A cohort of 184 stage I-III NSCLC patients, 
staged according to the International System of Staging for Lung Cancer, was randomly selected. Information on baseline demographics, clinicopathological characteristics and surgical approach was collected after review of clinical notes and histopathology reports. Outcome data, including peri-operative mortality and long-term survival, was updated prospectively. Patients' characteristics are detailed in Table 1. This study was approved by the St. James's Hospital Ethics Committee.

Generation and staining of TMAs. A 4- $\mu \mathrm{m}$ section was cut from formalin-fixed, paraffin-embedded tissue blocks from the 184 patients. This section was stained with hematoxylin-eosin for light microscopic examination by a pathologist (EK) to confirm areas of tumour. Three to four areas of tumour were marked on each section. Three cores, each $2 \mathrm{~mm}$ in diameter, were removed from each patient 'donor' block and inserted into a 'recipient' TMA block. A series of sections were cut from each TMA block, the first and last sections being stained with hematoxylin-eosin with tumour content confirmed by a pathologist. TMAs were assessed for FLIP and procaspase-8 expression as previously reported. $^{39}$

TMA machine scoring and analysis. Slides were scanned using an Aperio ScanScope CS (Aperio, San Diego, CA, USA) at a resolution of $\times 40$ using the objective $\times 40 / 0.75$ Plan Apo with a doubler and loaded onto the local drive for storage. The digitized TMA slides were imported into Definiens Tissue Studio, v2.1 (Definiens AG, Munich) for image analysis. The digital images were 'dearrayed' within Tissue Studio to generate individual core images with registered coordinates matching the original TMA layout. Twelve of the dearrayed cores were used in a training set to define a completely novel cytonuclear algorithm for the quantitative analysis of FLIP and procaspase-8 expression. An automated region recognition module was applied to the training set to initially identify and distinguish regions of tumour, stroma and background at a magnification of $\times 20$ (Figure 1a). Nuclear detection was subsequently carried out independently on both tumour and stroma. Thresholds were identified to distinguish between positive and negative nuclei expression for each protein using an image object information table. In addition, a nuclei filter was used to remove over-segmented nuclei. Cell simulation based on growing boundaries around the nuclei was used to model and identify the cytoplasmic compartment (Figure 1b). This allowed automatic identification of cytonuclear compartments, within which protein expression density was then calculated. Results were reviewed by an experienced pathologist (MS-T). Nuclear and cytoplasmic expression of FLIP and procaspase-8 were objectively measured automatically across all TMA cores. Statistical analyses were performed using GraphPad Prism version 5. Comparisons between matched tumour and stroma were analysed using Mann-Whitney non-parametric t-test. Correlations were assessed using Pearson's correlation coefficient method. Disease-free survival between the groups was compared using Kaplan-Meier method and log-rank (Mantel-Cox) analysis.

Cell lines and cell culture. H460, A549 and 34LU cell lines were obtained from ATCC (Teddington, UK) and maintained as previously described. ${ }^{7}$ All cell lines were regularly screened for presence of mycoplasma using the MycoAlert Mycoplasma Detection Kit (Lonza, Basel, Switzerland). Cisplatin-resistant and Bax/Bak-shRNA H460 cells ${ }^{15,18}$ were described previously.

Generation of stable overexpressing cell lines. FLIP coding region was PCR amplified and ligated into the pBABEpuro vector, according to standard protocols. Phoenix GP cells (Nolan Lab, Stanford University) were co-transfected with $4 \mu \mathrm{g}$ of the pBABEpuro vector and VSVG coat-protein plasmid. After $24 \mathrm{~h}$, the virus-containing media was removed, filtered and added to $\mathrm{H} 460$ cells; stable transfectants were selected in $1 \mu \mathrm{g} / \mathrm{ml}$ puromycin (Sigma).

Reagents. Vorinostat (SAHA), panobinostat (LBH-589), entinostat (MS-275) and birinapant (TL32711) were purchased from Selleck Chemicals (Newmarket, UK); ACY-775 was obtained from Acetylon Pharmaceuticals, Boston, MA, USA; cisplatin (Hospira, UK) was obtained from the Belfast City Hospital Pharmacy. Recombinant human TRAIL and Z-VAD-fmk were purchased from Calbiochem (Watford, UK) and MG132 (Z-Leu-Leu-Leu-Leu-al) from Sigma-Aldrich (Gillingham, Dorset, UK)

Western blotting and antibodies. Cells were harvested and lysed in RIPA buffer. Western blotting was carried out as previously described. ${ }^{8}$ FLIP
(NF6) and caspase-8 (12F5) antibodies were obtained from Alexis Biochemicals (San Diego, CA, USA), PARP antibody was from eBioscience (San Diego, CA, USA), $\beta$-actin, HDAC3 and HDAC6 were from Sigma-Aldrich. Antibodies for acetylated alpha-tubulin, acetylated histone $\mathrm{H} 4$, caspase-3, XIAP, CIAP1, BAX, BAK, BCL-2, MCL-1, BID, HDAC1 and HDAC2 were from Cell Signaling Technology (Hitchin, UK)

Clonogenic assays. $\mathrm{H} 460$ and A549 cells were seeded at 500 cells per well on six-well plates. The following day, cells were treated as required. At the end of treatment, the media was replaced with fresh medium and allowed to grow until colonies formed. Cells were fixed in ice-cold methanol, stained with crystal violet solution and colonies counted.

MTT cell viability assays. Cells (4000) were seeded per well on 96-well plates. After $24 \mathrm{~h}$, cells were treated for the appropriate time, after which MTT $(0.5 \mathrm{mg} / \mathrm{ml})$ was added to each well and the cells incubated at $37^{\circ} \mathrm{C}$ for a further $2 \mathrm{~h}$. The culture medium was removed and formazan crystals reabsorbed in $200 \mu \mathrm{l}$ DMSO. Cell viability was determined by reading each well at $570 \mathrm{~nm}$ on a microplate reader (Molecular Devices, Wokingham, UK).

siRNA transfections. Scrambled control (SC) siRNA, FLIP, caspase-8, DR4, DR5 and Fas siRNAs were obtained from Dharmacon (Chicago, IL, USA) as previously described. ${ }^{40}$ siRNA transfections were carried out using OligofectAMINE (Invitrogen), according to the manufacturer's instructions.

Quantitative PCR (Q-PCR). RNA was isolated from harvested cells using GeneJET RNA purification columns (Thermo Scientific, St. Leon-Rot, Germany) and reverse transcribed using Moloney murine leukaemia virus-based reverse transcriptase kit (Invitrogen). Q-PCR analysis of FLIP gene expression was performed using Real Time Ready probes and the Roche LightCycler 480 system (Roche Diagnostics, Burgess Hill, UK).

Flow cytometry. Cell death was determined as previously described. ${ }^{8}$ Harvested cells were stained with propidium iodide (Sigma-Aldrich) and their DNA content evaluated on a BD FACS Calibur flow cytometer (BD, Oxford, UK).

Caspase activity assays. A volume of $25 \mu \mathrm{l}$ Caspase- 8 or caspase-3/7-GLO reagent (Promega, Southampton, UK) were added to $1-10 \mu \mathrm{g}$ of protein lysate and incubated for $1 \mathrm{~h}$ at room temperature. Luciferase activity was determined using a luminometer

Statistics for in vitro analyses. Student's t-test and two-way analysis of variance (ANOVA) were used for statistical analysis; * denotes $P<0.05$; ${ }^{* *}$ denotes $P<0.01 ;{ }^{* * *}$ denotes $P<0.001$. Drug interactions were determined by calculating $\mathrm{Cl}$ values calculated from isobolograms generated using the CalcuSyn software. A Cl value of $0.85-0.9$ is slightly synergistic, $0.7-0.85$ is moderately synergistic, $0.3-0.7$ is synergistic and $0.1-0.3$ is strongly synergistic. ${ }^{41}$

\section{Conflict of Interest}

Professor DA Fennell has worked as a consultant for Merck, who manufacture Vorinostat. The other authors declare no conflict of interest.

Acknowledgements. JSR was supported by a studentship from the Department of Education and Learning, Northern Ireland. NC and IS were supported by grants from the British Lung Foundation. CH and SVS were supported by CRUK.

1. Shirley S, Micheau O. Targeting c-FLIP in cancer. Cancer Lett 2013; 332: 141-150.

2. Micheau O, Tschopp J. Induction of TNF receptor I-mediated apoptosis via two sequential signaling complexes. Cell 2003; 114: 181-190

3. Tenev $\mathrm{T}$, Bianchi $\mathrm{K}$, Darding $\mathrm{M}$, Broemer $\mathrm{M}$, Langlais $\mathrm{C}$, Wallberg $\mathrm{F}$ et al. The Ripoptosome, a signaling platform that assembles in response to genotoxic stress and loss of IAPs. Mol Cell 2011; 43: 432-448.

4. Bots M, Johnstone RW. Rational combinations using HDAC inhibitors. Clin Cancer Res 2009; 15: 3970-3977. 
5. Spierings DC, de Vries EG, Timens W, Groen HJ, Boezen HM, de Jong S. Expression of TRAIL and TRAIL death receptors in stage III non-small cell lung cancer tumors. Clin Cancer Res 2003; 9: 3397-3405.

6. den Hollander MW, Gietema JA, de Jong S, Walenkamp AM, Reyners AK, Oldenhuis CN et al. Translating TRAlL-receptor targeting agents to the clinic. Cancer Lett 2013; 332: 194-201.

7. Wilson TR, Redmond KM, McLaughlin KM, Crawford N, Gately K, O'Byrne K et al. Procaspase 8 overexpression in non-small-cell lung cancer promotes apoptosis induced by FLIP silencing. Cell Death Differ 2009; 16: 1352-1361.

8. Kerr E, Holohan C, McLaughlin KM, Majkut J, Dolan S, Redmond K et al. Identification of an acetylation-dependant Ku70/FLIP complex that regulates FLIP expression and HDAC inhibitor-induced apoptosis. Cell Death Differ 2012; 19: 1317-1327.

9. Bangert A, Cristofanon S, Eckhardt I, Abhari BA, Kolodziej S, Hacker S et al. Histone deacetylase inhibitors sensitize glioblastoma cells to TRAIL-induced apoptosis by c-myc-mediated downregulation of cFLIP. Oncogene 2012; 31: 4677-4688.

10. Hurwitz JL, Stasik I, Kerr EM, Holohan C, Redmond KM, McLaughlin KM et al. Vorinostat/ SAHA-induced apoptosis in malignant mesothelioma is FLIP/caspase 8-dependent and HR23B-independent. Eur J Cancer 2012; 48: 1096-1107.

11. Paul I, Chacko AD, Stasik I, Busacca S, Crawford N, McCoy F et al. Acquired differential regulation of caspase-8 in cisplatin-resistant non-small-cell lung cancer. Cell Death Dis 2012; 3: e449.

12. Belani CP, Langer C. First-line chemotherapy for NSCLC: an overview of relevant trials. Lung Cancer 2002; 38(Suppl 4): 13-19.

13. Schiller JH, Harrington D, Belani CP, Langer C, Sandler A, Krook J et al. Comparison of four chemotherapy regimens for advanced non-small-cell lung cancer. N Engl J Med 2002; 346: 92-98.

14. Crawford N, Chacko AD, Savage KI, McCoy F, Redmond K, Longley DB et al. Platinum resistant cancer cells conserve sensitivity to $\mathrm{BH} 3$ domains and obatoclax induced mitochondrial apoptosis. Apoptosis 2011; 16: 311-320.

15. Bradner JE, West N, Grachan ML, Greenberg EF, Haggarty SJ, Warnow T et al. Chemical phylogenetics of histone deacetylases. Nat Chem Biol 2010; 6: 238-243.

16. Dokmanovic M, Clarke C, Marks PA. Histone deacetylase inhibitors: overview and perspectives. Mol Cancer Res 2007; 5: 981-989.

17. McLornan D, Hay J, McLaughlin K, Holohan C, Burnett AK, Hills RK et al. Prognostic and therapeutic relevance of C-FLIP in acute myeloid leukaemia. Br J Haematol 2013; 160: 188-198.

18. Ashkenazi A. Directing cancer cells to self-destruct with pro-apoptotic receptor agonists. Nat Rev Drug Discov 2008; 7: 1001-1012.

19. Fulda S, Vucic D, Targeting IAP. proteins for therapeutic intervention in cancer. Nat Rev Drug Discov 2012; 11: 109-124.

20. Juin P, Geneste O, Gautier F, Depil S, Campone M. Decoding and unlocking the BCL-2 dependency of cancer cells. Nat Rev Cancer 2013; 13: 455-465.

21. Letai AG. Diagnosing and exploiting cancer's addiction to blocks in apoptosis. Nat Rev Cancer 2008; 8: 121-132.

22. Shivapurkar N, Toyooka S, Eby MT, Huang CX, Sathyanarayana UG, Cunningham HT et al. Differential inactivation of caspase-8 in lung cancers. Cancer Biol Ther 2002; 1: 65-69.

23. Logan AE, Wilson TR, Fenning C, Cummins R, Kay E, Johnston PG et al. In vitro and in vivo characterisation of a novel c-FLIP-targeted antisense phosphorothioate oligonucleotide. Apoptosis 2010; 15: 1435-1443.

24. Frew AJ, Lindemann RK, Martin BP, Clarke CJ, Sharkey J, Anthony DA et al. Combination therapy of established cancer using a histone deacetylase inhibitor and a TRAIL receptor agonist. Proc Natl Acad Sci USA 2008; 105: 11317-11322.

25. Schuchmann M, Schulze-Bergkamen H, Fleischer B, Schattenberg JM, Siebler J, Weinmann $A$ et al. Histone deacetylase inhibition by valproic acid down-regulates
c-FLIP/CASH and sensitizes hepatoma cells towards CD95- and TRAIL receptor-mediated apoptosis and chemotherapy. Oncol Rep 2006; 15: 227-230.

26. Pathil A, Armeanu S, Venturelli S, Mascagni P, Weiss TS, Gregor M et al. HDAC inhibitor treatment of hepatoma cells induces both TRAIL-independent apoptosis and restoration of sensitivity to TRAIL. Hepatology 2006; 43: 425-434.

27. Rao-Bindal K, Koshkina NV, Stewart J, Kleinerman ES. The histone deacetylase inhibitor, MS-275 (entinostat), downregulates c-FLIP, sensitizes osteosarcoma cells to FasL, and induces the regression of osteosarcoma lung metastases. Curr Cancer Drug Targets 2013; 13: $411-422$.

28. Dickinson M, Johnstone RW, Prince HM. Histone deacetylase inhibitors: potential targets responsible for their anti-cancer effect. Invest New Drugs 2010; 28(Suppl 1): S3-20.

29. Minucci S, Pelicci PG. Histone deacetylase inhibitors and the promise of epigenetic (and more) treatments for cancer. Nat Rev Cancer 2006; 6: 38-51.

30. Xu WS, Parmigiani RB, Marks PA. Histone deacetylase inhibitors: molecular mechanisms of action. Oncogene 2007; 26: 5541-5552.

31. Frew AJ, Johnstone RW, Bolden JE. Enhancing the apoptotic and therapeutic effects of HDAC inhibitors. Cancer Lett 2009; 280: 125-133.

32. Kelly WK, O'Connor OA, Krug LM, Chiao JH, Heaney M, Curley T et al. Phase I study of an oral histone deacetylase inhibitor, suberoylanilide hydroxamic acid, in patients with advanced cancer. J Clin Oncol 2005; 23: 3923-3931.

33. Blumenschein GR Jr., Kies MS, Papadimitrakopoulou VA, Lu C, Kumar AJ, Ricker JL et al. Phase II trial of the histone deacetylase inhibitor vorinostat (Zolinza, suberoylanilide hydroxamic acid, SAHA) in patients with recurrent and/or metastatic head and neck cancer. Invest New Drugs 2008; 26: 81-87.

34. Kelly WK, Richon VM, O'Connor O, Curley T, MacGregor-Curtelli B, Tong W et al. Phase I clinical trial of histone deacetylase inhibitor: suberoylanilide hydroxamic acid administered intravenously. Clin Cancer Res 2003; 9(10 Pt 1): 3578-3588.

35. Kavanaugh SM, White LA, Kolesar JM. Vorinostat: A novel therapy for the treatment of cutaneous T-cell lymphoma. Am J Health Syst Pharm 2010; 67: 793-797.

36. Knipstein J, Gore L. Entinostat for treatment of solid tumors and hematologic malignancies. Expert Opin Investig Drugs 2011; 20: 1455-1467.

37. Ramalingam SS, Maitland ML, Frankel P, Argiris AE, Koczywas M, Gitlitz B et al. Carboplatin and Paclitaxel in combination with either vorinostat or placebo for first-line therapy of advanced non-small-cell lung cancer. J Clin Oncol 2010; 28: 56-62.

38. Johnstone RW, Frew AJ, Smyth MJ. The TRAIL apoptotic pathway in cancer onset, progression and therapy. Nat Rev Cancer 2008; 8: 782-798.

39. McLornan DP, Barrett HL, Cummins R, McDermott U, McDowell C, Conlon SJ et al. Prognostic significance of TRAIL signaling molecules in stage II and III colorectal cancer. Clin Cancer Res 2010; 16: 3442-3451.

40. Wilson TR, McLaughlin KM, McEwan M, Sakai H, Rogers KM, Redmond KM et al. C-FLIP: a key regulator of colorectal cancer cell death. Cancer Res 2007; 67: 5754-5762.

41. Chou TC, Talalay P. Quantitative analysis of dose-effect relationships: the combined effects of multiple drugs or enzyme inhibitors. Adv Enzyme Regul 1984; 22. 27-55.

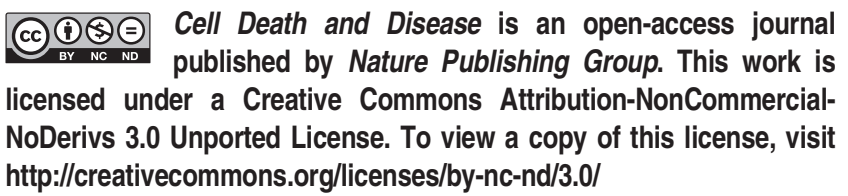
http://creativecommons.org/licenses/by-nc-nd/3.0/

Supplementary Information accompanies this paper on Cell Death and Disease website (http://www.nature.com/cddis) 